\title{
Equilibración cognitiva y equilibrio cognitivo: la perspectiva de la Escuela de Ginebra
}

\author{
J. M. Serrano \\ J. A. Carranza \\ A. G. BRITO \\ Universidad de Murcia

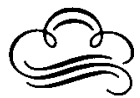

\section{Resumen}

Se estudia la evolución de las formas de equilibración cognitiva utilizadas en la Escuela de Ginebra. Después de hacer un análisis sobre el papel de la asimilación-acomodación en las formas posibles de equilibración cognitiva dentro del marco de las ciencias fácticas, se extraen las propiedades que son exclusivamente caracteristicos de aquéllas. A continuación, se define el proceso de adaptación como el equilibrio entre sus dos polos (asimilativo y acomodativo), incidiendo sobre la iaturaleza interactiva de éstos, caracterizando el equilibrio por las conservaciones recíprocas de los procesos y mostrando cómo estas conservaciones podrian considerarse bajo tres dimensiones que conducirian a tres formas, cualitativamente diferentes, de equilibración cognitiva. Finalmente, con el deslizamiento del interés desde las estructuras a los procedimientos se produce una modificación en la conceptualización del equilibrio, que muestra las nuevas posibilidades de equilibración de los esquemas cognitivos (presentativos, procedimientales $y$ operatorios).

\section{Abstract}

The evolution of cognitive equilibration used in the School of Geneve is studied. After analyzing the role of assimilation/accommodation in the possible forms of equilibration within the framework of factic sciences, the properties that exclusively belong to the cognitive equlibration are put forward. Next, it is defined the adaptative process as the equilibrium between its assimilative and accommodative poles, emphasizing the interactive nature of assimilation and accommodatios. The equlibrium is featured by the reciprocal conservations of the processes, and it is shown bow these conservations can be considered under three aspects. Each aspect should lead to a qualitatively different cognitive equilibration. Finally, with the sliding of the interest from the estructures to the procedures within the School of Geneve, there is a change in the conceptualization of equilibrium. As a consequence of it, the new possibilities of equilibration of cognitive schemes (presentatives, procedural and operational) are shown.

Dirección de los autores: Departamento de Psicología Evolutiva y de la Educación. Universidad de Murcia. Campus de Espinardo. 30071 Murcia. 


\section{4}

\section{EQUILIBRACION COGNITIVA Y EQUILIBRIO COGNITIVO: LA PERSPECTIVA DE LA ESCUELA DE GINEBRA}

La teoría epistemológica de Piaget nos lleva a una posible inclusión de la génesis de lo psicológico en la génesis de lo vital. Así, cualquier estructura biológica podría extenderse más allá de su propia corporalidad espacio-temporal. Esto implica, por un lado, reconocer que el comportamiento es el verdadero motor de la evolución (Piaget, 1977a, págs. 11 y ss.) y, por otro, la consideración, producto de esa evolución, de las estructuras cognitivas como órganos intelectuales. Su formación, ontogenéticamente, está en función del propio sistema epigenético, esto es, que depende totalmente de tres grandes factores: (a) las características de un programa inicial inscrito en el genoma: herencia: (b) las condiciones particulares de existencia: intercambios con el medio; y (c) un centro funcional que, a modo de compilador y CPU (Unidad central de procesamiento) de un ordenador, haga posible leer y realizar el programa: funcionamiento.

Sin embargo, y a fin de que se produzca un desarrollo coherente, tanto desde el plano biológico como del psicológico, estos tres factores necesitan «actuar» de forma coordinada, para lo que se precisa la concurrencia de un cuarto factor que mantenga la armonía del sistema: la autorregulación.

Por esta razón, el concepto de equilibrio llega a ser una propiedad constitutiva de la vida misma, un proceso constructivista generador de estructuras cognitivas, y la mejor expresión de la ley funcional que afirma la propia actividad estructural. No puede extrañar, pues, que la noción de equilibrio se haya mantenido, desde el comienzo, como uno de los conceptos crucialse en la teoría de Piaget, y que la idea de elaborar una teoría satisfactoria de los procesos de equilibrio (teoría de la equilibración) fuera una constante y un norte en su vida y su obra que nunca llegó a satisfacerle totalmente (Piaget, 1977a).

\section{TRES FORMAS DE CONCEPTUALIZAR EL EQUILIBRIO}

En la escala tradicional de las ciencias nomotéticas fácticas, la Psicología ocupa el tercer lugar de la misma, tras las Ciencias Físicas y las Naturales (o Biológicas). Al tomar prestado el concepto de equilibrio de las dos primeras, hemos de establecer primero las diferencias y similitudes que permitan aclarar el término desde este nuevo punto de vista, es decir, hemos de redefinir el equilibrio para adaptarlo a su nueva acepción.

Si comparamos los equilibrios físicos y cognitivos, observamos que ambos presentan una característica común: la reversibilidad. La propia evolución de los rasgos definidores del concepto en la teoría de Piaget, hasta llegar al carácter de reversibilidad como rasgo fundamental del equilibrio, nos servirá para acercarnos a la comprensión de la base bio-física del concepto y, finalmente, para situarlo dentro del marco cognitivo.

En un debate sobre «Nociones de estructura y génesis» dirigido por De Candillac, Goldmann y Piaget (Piaget, 1966), éste definió como caracteres comunes a todo equilibrio: la estabilidad, la compensación y la actividad; siendo la primera «cinética» o «cinemática», es decir, no inmóvil; indicando la segunda cualquier forma de reducción de una perturbación; y la tercera, que el trabajo realizado es proporcional a la fuerza estructurante.

El carácter «cinético» o «cinemático» del equilibrio se aprecia cuando se juega una partida de ajedrez, por ejemplo, en el permanente equilibrio de las piezas 


\section{5}

sobre el tablero. A cada par de jugadas (blancas/negras) sigue un equlibrio (generalmente no simétrico, pero perfectamente observable incluso para un profano en las técnicas del juego) que al sufrir constantes cambios a lo largo de su desarrollo se puede considerar cinético o estático.

El carácter compensatorio del equilibrio se puede observar en los sujetos que, ante una prueba de conservación de elementos discretos (la célebre prueba de las fichas, por ejemplo), y tras el desplazamiento de los mismos, deshaciendo la igualdad (correspondencia óptica u operatoria) por ellos establecida, elaboran un mecanismo compensatorio (operatorio o no) que anule la perturbación introducida, mediante una adición o sustracción de unidades que le permita mantener la igualdad -al menos en un aspecto perceptivo-espacial-, o bien por el desplazamiento inverso al efectuado sobre los elementos, lo que supone una anulación de la acción en el plano de la acción (reversibilidad empírica o retorno a la situación inicial), o bien por anulación de la acción en el plano del pensamiento (reversibilidad operatoria): «no se han añadido o quitado fichas, luego siguen habiendo las mismas» (Serrano, 1982).

Por último, el rasgo de actividad vendría a expresar que la mayor capacidad de manipulación de los objetos (y sus propiedades) estaría en función de las posibilidades de interacción entre las estructuras que componen el sistema (o subsistema). En efecto, un sistema con mayor capacidad diferenciadora es capaz de poner en juego mayor número de estructuras y, por tanto, al analizar la realidad, su mayor poder estructurante le permite un mayor rendimiento. En nuestro ejemplo anterior, como el sujeto inicialmente sólo pone en juego una estructura topológica o espacial, las posibilidades de compensación son muy escasas y sólo alcanza a mantener una igualadad perceptivo-espacial. Cuando otras estructuras (numéricas, de clasificación, de relaciones asimétricas, etc.) enriquecen el sistema de cuantificación lógico-matemática, el sujeto realiza, de forma progresiva, un mejor análisis de la realidad, gracias al descubrimiento de propiedades inherentes a la cantidad, distintas a las puramente espaciales. Es decir, al aumentar la «fuerza estructurante» del sistema, su aplicación a lo real, le permite realizar una mayor «trabajo» sobre la misma, que se manifiesta especialmente en la elaboración de nuevas formas de análisis.

A. Moles cuestionó el reduccionismo que aparentemente surgía entre los dos últimos factores. Piaget lo aceptó y redujo los tres «aspectos» por él mencionados a dos caracteres básicos: la estabilidad y la compensación, caracteres que ha venido manteniendo hasta 1974. A partir de entonces, los sintetiza en uno, ya mencionado anteriormente: la reversibilidad (Piaget, 1978a; Piaget, 1978b, por ejemplo).

Ahora bien, salvo las moderaciones a las perturbaciones realizadas por y a través de un desplazamiento, en un equilibrio físico no se producen modificaciones. En este sentido, desde el punto de vista de la Estática, la condición de equilibrio de un sólido rígido, con velocidad nula, es la anulación de la resultante de todas las fuerzas exteriores que actúan sobre él. Así, tras dar al sistema un desplazamiento (perturbación), puede que el sólido, por un lado, tienda a recuperar la acción previa que suponemos era de equilibrio (equilibrio estable); por otro, que no recupere la posición inicial de equilibrio (equilibrio inestable); o finalmente, que no presente tendencia alguna a recuperarla o separarse de ella (equilibrio indiferente). Supongamos un cono apoyado sobre su base (Figura 1); el centro de gravedad (c.g.) cae en el centro de la base de sustentación. Si desplazamos lateralmente el cono de forma que el c.g. quede dentro de los límites 
de la base de sustentación, al cesar la fuerza del desplazamiento, el cono recobra la posición de su equilibrio inicial, esto es, de equilibrio estable. Si la fuerza aplicada origina un desplazamiento hacia fuera de los límites de la base de sustentación, el sólido no podría recuperar el equilibrio inicial, estando pues ante una posición de equilibrio inestable. Si, por último, el cono se apoya sobre su generatriz, y aplicamos una fuerza, el desplazamiento no presenta tendencia alguna a separarse o recuperar su posición inicial, dándose lo que se denomina equilibrio indiferente.

\section{FIGURA 1}

Ejemplo de los diversos tipos de equilibrio físico con una figura geométrica
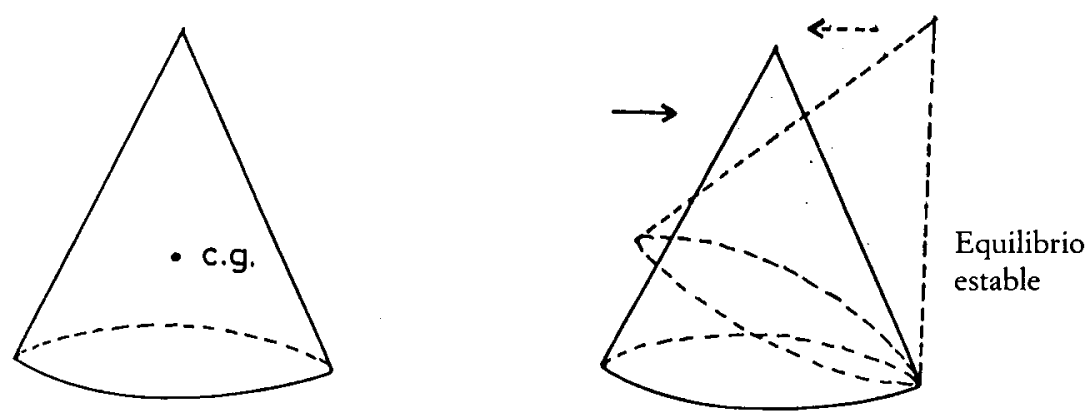

Equilibrio

inestable
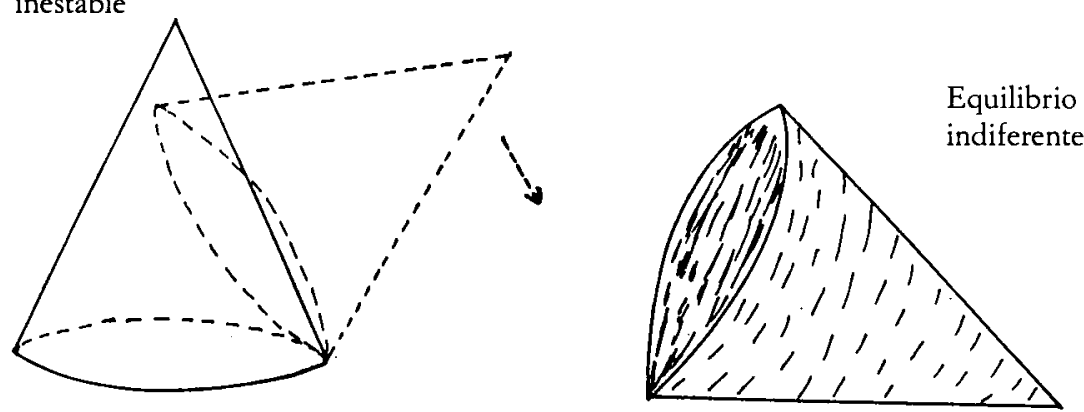

Igualmente, desde el punto de vista de la Dinámica, si al sólido no se le añade la condición de velocidad nula, se dice que estamos en condiciones de equilibrio dinámico. Supongamos que hacemos rodar una moneda y, al igual que en el caso del equilibrio estático, anulamos la resultante de todas las fuerzas de rozamiento (superficie, aire, etc.) que actúan sobre ella. La moneda entonces permanecería rodando con un movimiento constante y uniforme. Así pues, los aspectos estáticos y dinámicos del equilibrio físico ponen de relieve que los desequilibrios a que puede someterse un sólido dan lugar a un nuevo equilibrio, 
donde no se producen alteraciones en las propiedades que definen la estructura del mismo.

En Termodinámica, el equilibrio se produce por la transformación irreversible de las estructuras, alcanzando un nuevo estado integrado donde no es posible diferenciar las estructuras iniciales. Si, por ejemplo, se ponen en contacto dos cuerpos de temperatura diferente, el nuevo estado de equilibrio que surge, equilibrio térmico, resulta del flujo de calor del cuerpo de mayor temperatura al de menor. De acuerdo con el segundo principio de la termodinámica, el equilibrio es unidireccional, irreversible, por la imposibilidad de retorno al estado de las estructuras iniciales.

Las equilibraciones cognitivas, por el contrario, están basadas en las acciones conservadoras que los elementos, subsistemas y/o sistemas ejercen entre sí, y que se aplican al sistema total a través de los subsistemas o de sus elementos y viceversa. Por tanto, puede afirmarse que los equilibrios cognitivos se apoyan, entre otras cosas, en la solidaridad entre la diferenciación y la integración. Sirva de ejemplo un sistema cognitivo como el cuantificador lógico-matemático. La cuantificación puede tener carácter extensivo si permite establecer relaciones cuantificadoras ente la parte y el todo y entre las partes entre sí. Así mismo, podría tener carácter intensivo cuando sólo es posible establecer relaciones entre la parte y el todo. Siguiendo con el ejemplo, supongamos la descomposición del número 7 en dos sumandos: 5 y 2. La adición de estos dos elementos compondrían nuestro todo $(5+2=7)$. Esto nos permite comparar cada una de las partes con el todo $(5 \leq 7$ y $2 \leq 7)$, pero también permite la comparación de las partes entre sí ( $2 \leq 5)$. Respecto al carácter intensivo, tomemos como ejemplo un conjunto de flores $(\mathrm{F})$ formado por rosas $(\mathrm{R})$ y claveles $(\mathrm{C})$. La adición lógica $\mathrm{R}+\mathrm{C}$ sería igual a $F$. Esta adición permite una cuantificación en la que se establecen las relaciones de parte a todo ya mencionadas $(\mathrm{R} \leq \mathrm{F}$ y $\mathrm{C} \leq \mathrm{F})$, pero no permite establecer relación alguna entre las partes de ltipo $C \leq R$ ó $R \leq C$ ), pues desconocemos si $\mathrm{R} \leq \mathrm{C}$, ó $\mathrm{R} \leq \mathrm{C}$; en cambio, sabemos que el número de flores siempre será mayor (o igual, si R ó $C$ son $\varnothing$ ) que el de las rosas o el de claveles. El sistema general de cuantificación de los objetos, pues, se ha descompuesto en dos subsistemas: el de cuantificación extensiva y el de cuantificación intensiva.

Para operar con el subsistema de cuantificación intensiva, los sujetos deben poseer dos estructuras: de clase y relaciones asimétricas. Ambas están sujetas a sistemas de encaje jerárquico en función de las relaciones que cualifican o diferencian los elementos. Así, en nuestro ejemplo, los encajes jerárquicos $\mathrm{R} \leq \mathrm{F}$ y $C \leq F$ hacen referencia a las cualidades de los elementos ya que $\mathrm{R}$ y $\mathrm{C}$ se pueden reunir porque se parecen en $\mathrm{F}$ ( $\mathrm{R}$ y $\mathrm{C}$ son flores). En este subsistema la cuantifiación surgiría del uso de los cuantificadores intensivos «todos», «alguno» $\mathrm{O}$ «ninguno» (todos los $\mathrm{R}$ son $\mathrm{F}$, todos los $\mathrm{C}$ son $\mathrm{F}$, algún $\mathrm{F}$ es $\mathrm{R}$, algún $\mathrm{F}$ es $\mathrm{C}$, ningún $\mathrm{R}$ es $\mathrm{C}$ ). Si añadimos un nuevo elemento (por ejemplo, $\mathrm{P}=$ perros) en el sistema, el sujeto puede rechazarlo al no ser capaz de establecer un nuevo encaje jerárquico (seres vivos $=$ perros + flores) - sin que ello signifique que el sujeto no posea la estructura- o, al encontrar una cualidad que permite relacionar las flores con los perros, establecer un nuevo encaje ( $\mathrm{S}=$ seres vivos), cuantificando la nueva jerarquía bajo la forma «todos los los $\mathrm{P}$ son $\mathrm{S}$ », «todos los $F$ son $P$ », «algún $S$ es $P$ », «algún $S$ es $F$ » $y$ «ningún $P$ es $F$ ». En definitiva, la cuantificación intensiva supone establecer relaciones cualificadas.

Sin embargo, operar con el subsistema de cuantificación extensiva supone poseer una estructura numérica. En ella, las relaciones que se establecen no es- 
tán sujetas a la restricción anterior porque se producen entre elementos «cualesquiera», libres de las relaciones que lo cualifican o lo diferencian; es decir, las relaciones dentro de una estructura numérica pueden concebirse como un sistema de aplicaciones, libre de los encajes diferenciadores a los que están sometidas las estructuras de clases y relaciones. En nuestro caso númérico, descomponer 7 en los sumandos 5 y 2 no implica referencia alguna a cualidad; e incorporar un nuevo elemento, el 3 por ejemplo, no origina un nuevo encaje diferenciador, sino una nueva aplicación de las leyes de esa estructura al nuevo elemento $(5+2=7 ; 7+3=10)$. La cuantificación vendría determinada del mismo modo que en el caso inicial $(7 \leq 10 ; 3 \leq 10 ; 3 \leq 7)$. Por tanto, la cuantificación intensiva supone el establecimiento de relaciones «cualesquiera».

Estas estructuras se aplican a objetos reales o virtuales (elementos) sobre los que se establecen las leyes de cada una de ellas. Así, cuando un sujeto aplica las leyes de un subsistema o estructura de cuantificación lógico-matemática a determinadas propiedades de un objeto, dicha estructura entra en funcionamiento, al tiempo que pone en funcionamiento otras estructuras con las que interactúa, y todas ellas se aplican sobre un sistema integrador. En nuestro ejemplo de las flores, cuando un sujeto dice que «hay más rosas que claveles porque hay siete rosas y cinco claveles», su juicio refiere a una clase abarcadora (flores), a una relación asimétrica (más rosas que claveles) y a una evaluación numérica, al comparar las partes entre sí, haciendo abstracción de las cualidades «rosa» y «clavel». Queda claro el carácter integrador del sistema de cuantificación sin que ello, en ningún momento, vaya a menoscabo de la identidad de cada una de las estructuras. En otras palabras, las leyes de composición de cada estructura se aplican a un todo coherente sin perder su carácter diferenciador.

Desde esta perspectiva, y volviendo a las relaciones del equilibrio cognitivo con los equilibrios físico y biológico, las equilibraciones cognitivas están mucho más próximas a los conceptos biológicos de equilibración homeostática de Cannon (Piaget, 1971; Waddington, 1966, 1969) y homeorrésica (Waddington, 1969). «La homeostasis se emplea en conexión con sistemas que mantienen alguna variable como valor estable cuando pasa el tiempo... Empleamos la palabra homeorresis cuando lo que se estabiliza no es un valor constante sino un curso particular de cambio en el tiempo. Si algo sucede que altera un sistema homeorrésico los mecanismos de control no lo devuelve a donde habría llegado en algún momento posterior. La parte «resis» de la palabra deriva del griego Rbeo, fluir, y se puede concebir un sistema homeorrésico como algo bastante semejante a un río que corre por el fondo de un valle; si se produce un desprendimiento y saca el flujo del curso del valle, no lo devuelve al hecho en el lugar donde tuvo lugar el desvío, sino en algún lugar más alejado del mismo» (Waddington, 1969, p. 366). Se entiende, pues, por equlibrio homeosfático al permanente en el medio interno, que conduce a la autorregulación del organismo y considera la equilibración como estado; y por equilibrio homeorrésico al cinético del dèsarrollo que considera la equilibración como proceso. Otro ejemplo sería el del ciclo cardíaco, que está inmerso en un sistema más general de equilibrio biológico, donde se aprecia una evolución en los ritmos hasta alcanzar el del estado adulto. En el recién nacido el ritmo cardíaco es de 135-150 ciclos/minuto, ritmo que sufre modificaciones en el transcurso del desarrollo hasta estabilizarse en la edad adulta en torno a los 70-80 ciclos/minuto. Los diversos ritmos en esta evolución se pueden considerar estados o procesos. En el primer caso, podemos hablar de equilibrio estático, en tanto que durante períodos concretos el equili- 
brio lo determina un número concreto de ciclos. En el segundo, hablaríamos de equilibrio cinético, en tanto que hay una modificación del número de ciclos a fin de mantener el equilibrio en cada uno de los estados intermedios.

De igual manera, en el aspecto cognitivo, podemos considerar las distintas formas de equilibración cognitiva como estados o como procesos. Tomemos el ejemplo del niño que, tras una transformación (alargamiento) producida en una de dos bolas de plastilina, calificadas por él de idénticas en la cantidad inicial de materia que cada una contiene, nos dice que sigue existiendo igualdad entre las masas a pesar de la transformación. La forma de equilibrio del niño que se encuentra en este estado (conservación de la masa) es específica respecto a una determinada cantidad física. Si la propiedad a analizar fuera la de peso, podría darnos la misma respuesta u otra. En el primer caso, supondría que el niño presenta una forma más evolucionada de equilibrio (conservación del peso); en el segundo, no habría alcanzado aún esta nueva forma de equilibrio. Estas dos conservaciones podrían considerarse como estados (biológicamente corresponderían a equilibrios homeostáticos), o se podrían ver como un proceso de reequilibraciones sucesivas que llevan a los sujetos desde un estado — de conservación de la masa - a otro más evolucionado que el anterior - de conservación del peso-, (correspondiente al equilibrio homeorrésico en Biología) (Siegel, Bisanz y Bisanz, 1983, para mayor profundización).

No obstante, la conceptualización que hace Piaget del equilibrio como estado lleva implícita la asimilación recíproca de los conceptos de equilibrio y necesidad lógica. Cuando se alcanza el equilibrio, el niño se da cuenta de que la cantidad de plastilina, por ejemplo, es la misma a pesar de la transformación en la forma. Piaget afirma que, en este caso, un juicio correcto sobre la conservación de la cantidad se impone en sí mismo sobre el niño; es decir, que el juicio que ofrece el niño es inevitable, lógico o intrínsecamente necesario. La conclusión lógica del niño se ha alcanzado por equilibraciones sucesivas de manera que, como comentó un niño a Inhelder «una vez que uno sabe, uno sabe por siempre y para siempre» (Piaget, 1971b).

En la perspectiva biológica encontramos, asimismo, unas «leyes cinéticas específicas, que son las que permiten realizar el flujo de energía y de materia necesarios para construir y mantener un orden funcional y estructural en un sistema abierto» (Glansdorff y Prigogine, 1971, pág. 271). Del mismo modo, en el dominio cognitivo existen unas leyes «cinegéticas» estructurales, que permiten tanto la incorporación de elementos a la estructura como su transformación, que permiten mantener siempre un equilibrio en la estructura. Desde el plano biológico, el caso de la digestión obedece a unas leyes físico-químicas que permiten transformar la materia en energía, con el propósito de mantener el equilibrio en el organismo. En el plano cognitivo, cuando en el ejemplo de la pastilina, el niño respondía que al cantidad seguía invariante, se había reducido una asimilación de las características del estímulo según las leyes que componen la estructura. Esto supone, al mismo tiempo, haber considerado las características implícitas en la transformación de la materia. Así también, cuando el niño aplica las leyes estructurales de la conservación de la masa a la característica peso, para incorporar la nueva característica debe adecuar las leyes estructurales, de forma que cuando incorpora dicha característica se establece un nuevo equilibrio que permite mantener el orden funcional del sistema de evaluación de las cantidades físicas. Pero desde el marco psicogenético, los sistemas cognitivos no son totalmente abiertos, sino que, al igual que los sistemas biológicos, en 
tanto que «ciclos» pueden ser considerados en algún sentido como cerrados, y ésto, como veremos a continuación, debido a que los dos componentes del equilibrio (asimilación y acomodación) forman ya entre sí un ciclo. La acomodación, o la explicación del mundo físico, es un proceso centrífugo que se completa con el proceso centrípeto de la asimilación. La asimilación de un elemento a un sistema impone una acomodación, que implica la posibilidad de nuevas asimilaciones, lo que a su vez supone nuevas acomodaciones, y así sucesivamente, conformándose lo que denominamos ciclo cognitivo.

No obstante, los sistemas cognitivos pueden y deben ser considerados abiertos por sus posibilidades, que no necesidades, de intercambio con el entorno, siendo este segundo sentido el que los diferencia de los sistemas biológicos: éstos siempre necesitan contenidos exógenos para la elaboración de formas. Por el contrario, aunque la mayoría de los sistemas cognitivos se aplican a la realidad, existen sistemas formales donde el sujeto sólo usa la estructura primaria (con sus elementos) como objetos tematizados del pensamiento, elaborando nuevas forma a partir de otras formas y contenidos ya estructuradaso (operaciones intraproposicionales), esto es, elaborando nuevas formas a partir de contenidos endógenos. Cuando hablamos de un sujeto que posee un sistema de operaciones formales, ello supone aludir: 1) a la posibilidad de funcionamiento del sistema sin la necesidad de manipular elementos físicos, pudiendo ser los elementos estructurales ya elaboradas y 2) a la posibilidad de poder generar hipótesis a partir de estas estructuras. Por ejemplo, si sometemos a un sujeto a la prueba combinatoria de la E.P.L. se observa que no tiene necesidad de manipular los objetos concretos que se le dan (fichas de diversos colores) para dar la solución correcta al problema planteado. Supongamos que las dos primeras permutaciones binarias las ha resuelto. Cuando introducimos un nuevo elemento, no necesita establecer las seis permutaciones posibles, encontrándonos con respuestas del tipo «como hemos agregado un elemento que puede estar en tres posiciones distintas y antes tenía dos permutaciones posibles ahora tendré 2 por 3,6», y así sucesivamente responde ante las consecutivas adiciones de elementos.

En síntesis, como señala Bickhard (1988; Campbell y Bickhard, 1986), el equilibrio regulador es la propensión a compensar de forma coordinada perturbaciones potenciales, y el equilibrio perfecto, esto es, el equilibrio lógicomatemático o estructural, es la disposición a compensar de forma coordinada todas las perturbaciones posibles en un dominio. Dicho de otro modo, las estructuras lógico-matemáticas son cerradas, agotan algún dominio de metapotencialidades coordinadas, al contener todas las coordinaciones potenciales de dicho dominio, y por tanto, todas las vías posibles de reversibilidad. La equilibración puede considerarse así, como el proceso de construcción de organizaciones de coordinaciones progresivamente más cerradas, y donde se alcanza el cierre, punto en el que estas organizaciones se convierten en estructuras operacionales, la estructura llega a la necesidad lógico-matemática.

\section{EL EQUILIBRIO COGNITIVO: COMPONENTES BASICOS}

A partir de la alimentación y/o retroalimentación de los sistemas cognitivos, gracias a los contenidos endógenos y exógenos descritos más arriba, estos sistemas determinan unos ciclos epistémicos, cuya acción estructurante y estructurada (función), equivalente al carácter integrador y diferenciador ya vistos, y 


\section{1}

cuyo funcionamiento (actividad de la propia estructura ante la incorporación de algún elemento) se basan en dos procesos fundamentales que, como dijimos constituyen los componentes de todo equilibrio cognitivo: la asimilación y la acomodación.

Aunque los conceptos de asimilación y acomodación han sido tratados bajo todos los aspectos en Psicología (Piaget, 1969a, 1971, 1977a, 1977b, 1978a, 1978b; Flavell, 1978; Battro, 1971; Greco, 1959, 1960; Lacasa, 1982; cf. Vuyk, 1984 para una revisión), vamos a aclarar, y con fines de utilidad, estos dos polos de la adaptación, sin entrar en definiciones ortodoxas.

a) la asimilación es la incorporación de un elemento (esquema o estructura en calidades de subesquemas o subestructuras) a un esquema o estructura (sensoriomotor o conceptual) del sujeto. Las asimilaciones recíprocas son especialmente importantes, entendiendo este concepto en su sentido más amplio, es decir, en la aplicación a los mismos objetos de dos o más esquemas o subsistemas, la coordinación de los mismos sin necesidad de contenido real, y las relaciones entre un sistema total y los subsistemas que engloba en su diferenciación.

b) la acomodación es la necesidad con que se encuentra la asimilación de tener en cuenta las propias peculiaridades de aquellos que tiene que asimilar (elementos, esquemas, etc.). Esto lleva, de hecho, al planteamiento anterior sobre la asimilación, ya que si las asimilaciones recíprocas no estuviesen acompañadas de acomodaciones del mismo rango se originaría una fusión deformante (Piaget, 1978b), en lugar de una coordinación de los subsistemas.

\section{FigURA 2}

Diagrama descriptivo del praceso de asimilación

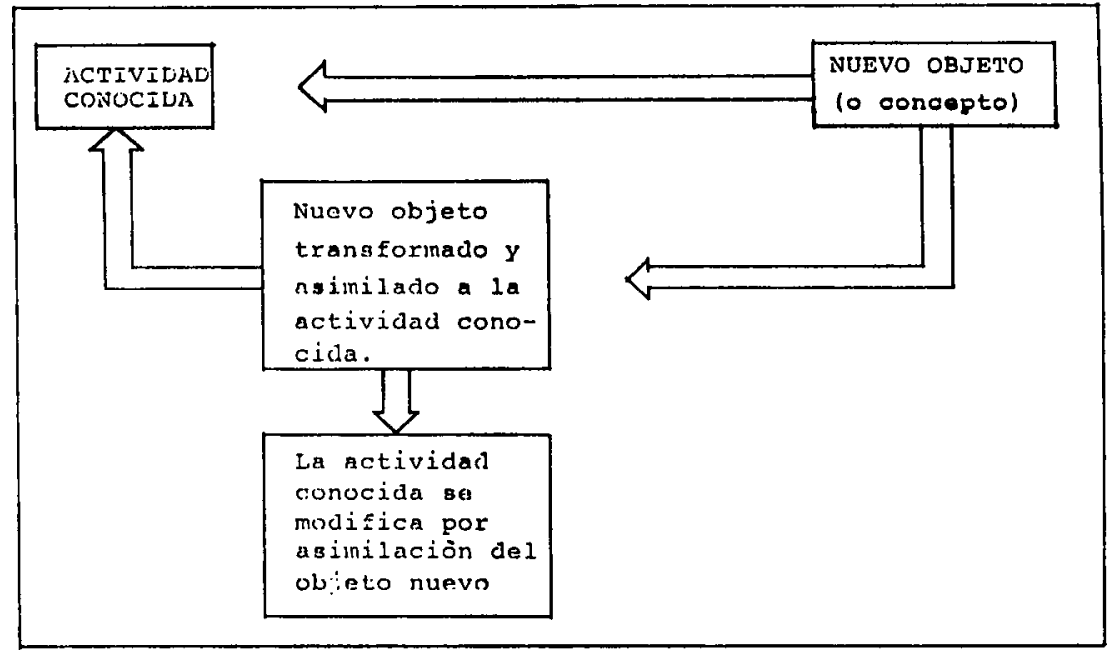

Planteada la cuestión en estos términos, la Escuela de Ginebra (Piaget, 1978b) recurre a dos hipótesis generales extraídas de los hechos, a saber: (1) Todo esquema de asimilación tiende a alimentarse (ver fig. 2), hipótesis que se limita a asignar un motor a la investigación; y (2) Todo esquema de asimilación se en- 
cuentra obligado a acomodarse en los elementos que asimila (ver fig. 3), hipótesis que afirma la necesidad de un equilibrio entre la asimilación y la acomodación.

FIGURA 3

Diagrama descriptivo del proceso de acomodación

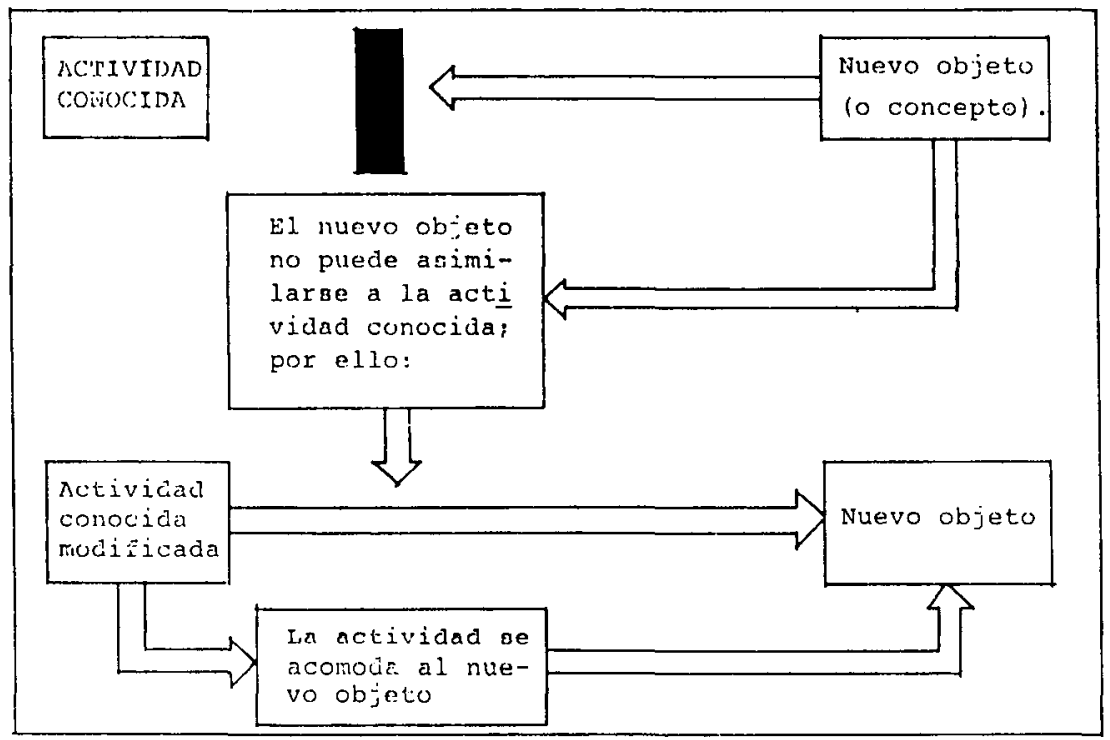

Aunque nos referimos a los dos procesos por separado, de hecho son los dos polos de todo proceso adaptativo; son, pues complementarios. Así, la adaptación supone el equilibrio entre la asimilación a la estructura y la acomodación de la estructura. Esto se constata en el modelo de equilibrio (modelo II de Piaget, 1978b):

$a$

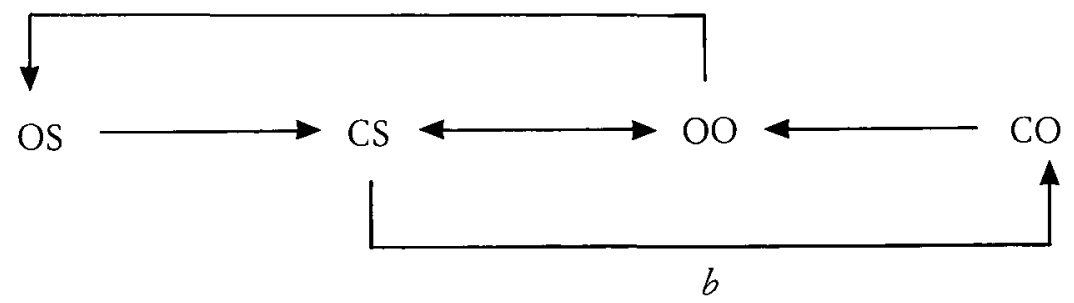

OS: observables en las acciones; OO: observables en el objeto; CS: aspectos inferenciales de las acciones; $C O$ : inferencias en los objetos; a: direcc. obj-sujeto (asimilación); b: direcc. suj.-objeto (acomodación); $\longleftrightarrow$ : equilibrio entre asimilación y acomodación. 
La descripción del funcionamiento del proceso de equilibrio es como sigue: descubrir deteminados observables en el objeto conduce al sujeto a descubrir algunos observables en su acción que, al coordinarse, posibilita el establecimiento de inferencias, hasta entonces ausentes en las propiedades de los objetos, con el siguiente descubrimiento de nuevos observables o significados de lo real, continuándose el ciclo hasta alcanzar el equilibrio definitivo.

\section{EQUILIBRIO COGNITIVO: FORMAS POSIBLES}

El equilibrio cognitivo queda definido, pues, por las conservaciones recíprocas de todo proceso adaptativo, que hasta ahora hemos considerado en tres vertientes:

En primer lugar, el sujeto se enfrenta a los objetos a través de sus esquemas de acción, siendo aquéllos los que ponen en marcha a éstos (asimilación; y los esquemas a su vez confieren significado a los objetos (acomodación), dándose el comienzo de conservación mutua y, por tanto, un equilibrio, un equilibrio entre asimilación y acomodación. Supongamos un sujeto acostumbrado a manipular objetos cuadrados al que le ponemos a su alcance un objeto esférico. $\mathrm{Al}$ aplicar el esquema que emplea para los primeros no puede cogerlo, produciéndose una modificación de dicho esquema, por ejemplo atrayéndolo hacia su cuerpo, que le sirve de soporte para poder cogerlo. La aplicación del esquema de «coger cosas cuadradas» para asimilar el objeto resultó inadecuado para su fin, lo que provoca unas acomodaciones a las características del nuevo objeto que culminan en la elaboración de otro esquema: «coger cosas esféricas».

En segundo lugar, citamos una equilibración entre subsistemas o subestructuras con carácter colateral, de jerarquía similar. Su construcción puede estar sujeta, de hecho lo está, a ritmos de formación diferentes, a desfases temporales más o menos notables, según las características de los objetos (estímulos) que el sujeto manipula en el plano de la acción y en el de la representación, pero donde finalmente se producirán las coordinaciones entre estructuras que llevarán a su mutua conservación.

En el ejemplo de la inclusión, podemos constatar la presencia de dos subsistemas con carácter colateral (cuantificación extensiva e intensiva) que interactúan, al mismo tiempo que se mantiene un equilibrio en las estructuras subyacentes (clases, relaciones y número). El proceso general de cuantificación es una asimilación recíproca de las estructuras de clases y relaciones a la de número, pues la estructura de número es, como vimos, indisociable de las estructuras lógicas y necesario para la consolidación de las mismas. Cuando el sujeto dice que hay más rosas que claveles porque las primeras son siete y las segundas cinco, hace abstracción de las cualidades (rosa y clavel) para comparar las partes entre sí (estructura numérica), sin olvidar que las rosas y los claveles estaban incluidos en la clase de las flores (estructura lógica).

Por último, citamos un equilibrio, el de las coordinaciones entre los subsistemas y la totalidad que los engloba, en sus vertientes diferenciadora e integradora. Este añade al anterior un carácter de jerarquización, donde «la integración, que es cuestión de asimilación, y la diferenciación que exige acomodaciones» (Piaget, 1978b, pág. 11) producirán, por equilibración entre ambas, la conservación mutua del todo y las partes.

Dentro del sistema de cuantificación lógico-matemático donde subyacen, entre otras, las estructuras mencionadas con anterioridad, podemos observar que, 
cuando al sujeto le planteamos un problema del tipo «¿Todas las flores son rosas?», y responde en términos de cuantificación intensiva ( No, porque también hay claveles»), esto es, en función de la estructura más adecuada de entre las que componen el sistema y al que están integradas, se ha dado una asimilación integradora de la estructura al sistema así como una acomodación diferenciadora del sistema: para resolver el problema ha elegido dicha estructura y no otra. En otras palabras, la respuesta da lugar al empleo de la estructura a la que pertenece la misma, siendo así asimilada al sistema del que forma parte.

\section{EQUILIBRACION PROCEDIMENTAL Y EQUILIBRIO}

Finalmente, en los años setenta surgen en la Escuela de Ginebra una serie de trabajos e investigaciones, latentes desde la década anterior que, con la introducción del par dialéctico: posible vs. necesario (Piaget, 1981, 1983), deslizan el estudio desde las estructuras a los procedimientos (Inhelder, 1978; Inhelder y Piaget, 1979; Piaget (1974). En consecuencia, tiene lugar un cambio teórico sustancial, al establecer dos sistemas complementarios en el seno de los mecanismos cognitivos del desarrollo. Así, vemos un ajuste sistemático de los modelos de equilibración biológica al nuevo marco psicológico de la epistemología genética.

El primer sistema, estructural y destinado a comprender el conjunto de los real, lo forman los esquemas presentativos y operatorios (estos últimos en tanto que estructuras). El segundo sistema, procedimental y destinado a logar el éxito en todos los dominios (desde la acción a la más pura abstracción), contiene esquemas procedimientales y operatorios (estos últimos en cuanto operaciones transformadoras encaminadas a solucionar problemas).

En este marco, son esquemas presentativos aquellos que versan sobre caracteres permanentes y simultáneos de objetos comparables, y lo constituyen los esquemas representativos (o conceptos) y sensoriomotores. La característica fundamental es la de poder ser generalizados y abstraídos de su contexto con facilidad. Los esquemas procedimentales, en cambio, son las consecuencias de acciones que crean medios para alcanzar su fin, siendo su característica fundamental la precursividad: la determinación de las acciones iniciales por la tendencia de las mismas hacia un estado ulterior.

En efecto, el segundo sistema, como todo procedimiento, consiste en un conjunto de estados de transición en la consecución de un objetivo que, una vez conseguido, hace inoperante el propio procedimiento. Esto es, deja de existir como tal logrado el objetivo. Así, toda operación psicológica es no sólo un concepto (atemporal) y, por tanto, solidaria de una estructura, sino además un artefacto (temporal) y, por ende, solidaria de un procedimiento.

En el ejemplo de clasificación hay, pues, que distinguir dos aspectos: 1) la operación como acto temporal, que permite reunir rosas y claveles en la clase total de las flores y la consiguiente movilidad cognitiva que le permite encontrar las clases de las rosas, o de los claveles, a partir de la clase abarcadora (flores). Sin embargo, este acto ya no existe, como tal, una vez realizado. Y 2) la operación en tanto que concepto ( + ó-), que está adscrita, en este caso, al primer sistema y por lo tanto solidaria de una estructura de agrupamiento aditivo de clases.

Lo que constituye aquí la novedad es la conceptualización del equilibrio en sus dos planos: homeorrésico y homeostático. El equilibrio homeorrésico coin- 
cidiría con el concepto de equilibrio procedimental. Para Piaget tendría carácter particular, más o menos momentáneo, y siempre tratando de alcanzar una estabilidad cognitiva fuerte: equilibrio homeostático o estructural.

Sirva como aclaración el equilibrio en algunas fases del desarrollo de los esquemas numéricos. La estructura general de número muestra en algunos puntos de su evolución determinados caracteres (leyes) que, como instrumento de asimilación de lo real, permiten catalogarlos de organizaciones de conductas fuertemente estructurales, que están a título de subestructura en el seno de la categoría general de número. Así, los esquemas aditivos que permiten la composición y descomposición unidimensional del número natural (entero positivo), por la movilidad de estos esquemas, y siguiendo el proceso teleonómico que implica el principio de economía del pensamiento (Serrano, Carranza y Roca, 1985), favorecen el establecimiento de «posibles» descomposiciones (diferenciaciones) y su integración «necesaria» en un sistema más amplio. Esto desemboca en la construcción de lo que serán los futuros esquemas multiplicativos.

Así, se considera en una nueva totalidad, por un lado, la posibilidad de generalización de los esquemas aditivos y, por otro, la posibilidad de intersección consigo mismo. Es decir, la movilidad creciente de los esquemas aditivos permitirán mútiples posibilidades de descomposición del número, por ejemplo, $6=5+1=4+2=2+2+2$, etc., algunas de las cuales admiten la elaboración de un proceso de simplificación $6=3 \times 2$ (tres veces dos). Esto, junto con la posibilidad de asimilación recíproca a los esquemas de clase (que también alcanzan un amplio grado de movilidad) da lugar (ver Tabla I) a la construcción de los esquemas multiplicativos:

TABLA I

Ejemplo de esquema multiplicativo

\begin{tabular}{|c|c|c|c|c|c|c|}
\hline \multirow{3}{*}{2} & & CUADRADOS & TRIANGULOS & CIRCULOS & \multirow{3}{*}{\multicolumn{2}{|c|}{$\begin{array}{c}\text { Clase de rojos } \\
\text { Clase de } \\
\text { azules }\end{array}$}} \\
\hline & ROJO & Cuad. rojo & Triang. rojo & Circ. rojo & & \\
\hline & \multirow[t]{3}{*}{ AZUL } & Cuad. azul & Triang. azul & Circ. azul & 3 & \\
\hline & & 2 & 2 & 2 & $6^{*}$ & \\
\hline & & $\begin{array}{l}\text { Clase de } \\
\text { Cuadrados }\end{array}$ & $\begin{array}{l}\text { Clase de } \\
\text { Triángulos }\end{array}$ & $\begin{array}{l}\text { Clase de } \\
\text { Círculos }\end{array}$ & & \\
\hline
\end{tabular}

No obstante, constituir esquemas multiplicativos en una estructura de conjunto no absorbe o elimina la estructura aditiva previa; antes bien, da lugar a una nueva construcción estructural que integrará como subestructura a los es- 
quemas aditivos (y sus leyes), y los esquemas multiplicativos (y las suyas) con sus relaciones (leyes de integración, p. ej., la distributividad).

Esto quiere decir que ante una situación problema, el nuevo sistema «decide» que subestructura pone en marcha el sujeto para «solucionar» la situación, estando las dos igualmente disponibles. Así, si preguntamos a los niños por el resultado de la operación $2 \times 3$, y se le hace ver que ese resultado coincide con $3+3$, nos responde en la linea de posibles equivalencias de ambas operaciones, sin descartar ninguna de las dos. Esto quiere decir que ha alcanzado dos formas de reversibilidad operatoria, dos estructuras acabadas, esto es, dos niveles-estados de equilibrio.

Sin embargo, en la construcción del sistema aditivo, y antes de alcanzar su propia reversibilidad operatoria, el sujeto emplea, en su intento de conseguirla, otras formas de reversibilidad momentáneas (retorno empírico, invertibilidad, p. ej.). En efecto, antes de alcanzar la estructura aditiva, observamos un punto en el desarrollo donde el niño responde a los problemas de reestablecimiento de la igualdad desde la perspectiva del retorno y está convencido de la validez de su juicio. Una vez alcanzada la reversibilidad, el niño se niega a admitir la posibilidad de reobtener la igualdad por medio de una conducta de retorno, e incluso niega haber podido dar alguna vez una respuesta de retorno ante dicha situación. Este retorno o reversibilidad empírica es lo que vendría a ser una situación de equilibrio-procedimiento.

En síntesis, el objetivo inicial de estudiar las estructuras per se, donde la formación de las operaciones estaba subordinada a un proceso general de equilibración se ha pasado a un interés por los procesos. Assí, a partir de los años 70 , esto se ha abordado en obras sobre el estudio de las explicaciones causales, la toma de conciencia, las formas de contradicción, la abstracción y la generalización, las correspondencias, los isomorfismos y categorías, la dialéctica y la lógica de las significaciones, y sobre todo, la subordinación de las operaciones a funcionamientos más amplios de apertura sobre lo posible y lo necesario. Así mismo, entran en juego las investigaciones sobre el aprendizaje operatorio y los mecanismos aquí citados de los procedimientos y estrategias.

\section{Referencias}

Battro, A.; (1971). El pensamiento de Jean Piaget. Buenos Aires: Emece Ed.

BickHARD, M. H. (1988). Piaget on variation and selection models: Structuralism, logical necessity, and interactivism. Human Development, 31, 274-312.

CampBell, R. y Bickhard, M. H. (1986). Knowing levels and developmental stages Basilea: Karger.

Flavell, J. H. (1962). The developmental psychology of Jean Piaget. Nueva Jersey: Van Nostrad. (Trad. cast. ed. Paidos, 1978).

Glansdorf, P. y Prigogine, I. (1971). Structure, stabilité et fluctuations. París; Masson ed.

GRECO, P. (1959). Lapprendissage dans une situation de structure operatoire concrete: les innversions sucesives de l'ordre lineaire par des rotations de 180 degrés. En Apprendissage et connaissance. París: P.U.F.

GRECO, P. (1960): Recherches sur quelques formes d'inferences arithmetiques et sur la comprehension de Linteration numerique chez l'enfant. en Problemes de la construction du nombre. París: P.U.F.

INHELDER, B. (1978). De l'approche structurale a l'approche procédurale; introduction à l'etude des strategies. Acta XXI'eme congrès international de Psychologie. París: P.U.F. 


\section{7}

Inhelder, B. y Piaget, J. (1979): Procedures et Structures. Archives de Psychologie, 30, 1-9.

LACASA, P. (1982). La actividad del sujeto en el proceso de equilibración de las estructuras cognitivas en Jean Piaget. Madrid: Ed. de la Univers. Complutense.

Piaget, J. (1957). Logique et equilibre dans les comportement du sujet. En Logique et equilibre. París: P.U.F.

PAGET, J. (1959). La formation du symbole bez l'enfant: Imitation, jeu et rêve. Image et representation. Neuchâtel-París: Delachaux-Niestlé. (Trad. cast. ed. Fondo de Cultura Económica, 1977a).

Piaget, J. (1961). La naissance de l'intelligence chez l'enfant. Neuchâtel, Delachaux. (Trad. cast. ed. Aguilar, 1969a).

Piaget, J. (1966). Génese et structure dans psychologie. En Les notions de génese et de structure. La Haya: Mouton. (Trad. cast. ed. Proteo, 1969).

PIAGET, J. (1967). Biologie et connissance, essai sur les relations entre les regulations organiques et les processus cognitifs. París: Gallimard. (Trad. cast. Siglo xxI, 1977b)

Piaget, J. (1971b). The theory of stages in cognitive development. En D. R. Green, M. P. Ford y G. B. Flamer (comps.: Measurement and Piaget. Nueva York: McGraw-Hill.

Piaget, J. (1968). Le structuralisme. París: P.U.F. (Trad. cast. ed. Protep, 1971).

Piaget, J. (1974). Recherches sur le contradiction. París: P.U.F. (trad. cast. ed. Siglo xxI, 1978a).

PlaGet, J. (1974). Adaptation vitale et psychologie de l'intelligence. Selection organique et phénocopie. París: P.U.F. (Trad. cast. ed. Siglo xxI, 1978c).

Piaget, J. (1975). L'equilibration des structures cognitives. Próbleme central du developpment. París: P.U.F. (Trad. cast. ed. Siglo XXI, (1978b).

Piaget, J. (1981). Le possible et le nécessaire. Vol. I: L'evolution des comp. possibles chez l'enfant. París: P.U.F.

Piaget, J. (1983). Le possible et le necéssaire. Vol. II: L'evolution dur comp. necéssaire chez l'enfant. París: P.U.F.

Piaget, J.; Goldmann, L. y De Cadillac, M. (1966). Les notions de genese et structure. La Haya: Mouton. (Trad. cast. ed. Proteo, 1969).

Piaget, J. E INHELder, B. (1959). La genése des structures logiques elementaires. Classifications et seriations. Neuchâtel: Delachaux et Niestlé. (Trad. cast. ed. Guadalupe, 1975).

SERRANO, J. M. (1982). Un estudio de validación convergente para la determinación de niveles genéticos en la adquisición del concepto de número. Tesis doctoral inédita. Universidad de Murcia.

Serrano, S. M.; Carrranza, J. A. y Roca. (1985). El papel de la transitividad y de las inferencias transitivas en la adquisición del concepto de número. En Varios: Estudis y Recerques. La Pedagogía operatoria hoy. Barcelona: Ayuntamiento de Barcelona, Servicio de Publicaciones.

Siegel, A. W.; Bisanz, J. y Bisanz, G. L. (1983). Developmental analysis. A Strategy for Study of Psychological Change. En D. Kühn y J. A. Meacham (Comps.: On the development of Developmental Psychology. Nueva York: Karger.

Travers, J. F. (1982). The growing child. Nueva York, Scott, Foreman.

Waddington, C. H. (1966). Prnciples of development and differentiation. Nueva York: McMillan.

Wadington, C. H. (1969). Towards a theoretical biology. Chicago, Aldine. 